\title{
Tilted Bulk Dusty Universe
}

\author{
Rakeshwar Purohit and Anita Bagora
}

\begin{abstract}
Bianchi type I bulk viscous fluid tilted cosmological model filled with dust fluid is investigated. We assume that $\zeta \theta=K$ (constant), where $\zeta$ is the coefficient of bulk viscosity and $\theta$ is the expansion in the model. It has been assumed that the universe is filled with dust together with $\mathrm{A}=$ BC, where A, B and C are metric potentials. Special model is also investigated in the absence of bulk viscosity. The physical and geometrical aspects of the model in the presence and absence of bulk viscosity are also discussed.
\end{abstract}

Index Terms - Tilted cosmological model, bulk viscosity, bianchi type-I universe, dust fluid.

\section{INTRODUCTION}

Anisotropic cosmological models play an important role in the description of the universe in its early stage of evolution. Homogeneous and anisotropic models are called Bianchi Type I - IX [1]. There are two major cases arise: Orthogonal universe in which the matter moves orthogonally to the hyper surfaces of homogeneity and tilted universe in which the fluid is not normal to the hyper surfaces [2]. In recent years there has been a considerable interest in spatially homogeneous and anisotropic universe in which the fluid flow is tilted. These universe necessarily appear inhomogeneous to an observer. King and Ellis [2] have shown that time variation in pressure would cause the fluid to experience a spatial pressure gradient which made it more non-geodesic curves and the fluid can move with nonzero vorticity in general. King and Ellis [2] have found that there is no Bianchi type I tilted models if it has been obtained under the assumption that the matter takes the perfect fluid form:

$$
T_{i j}=(\in+p) v_{i} v_{j}+p g_{i}^{j}, \quad v_{i} v_{j}=-1, \in>0, p>0
$$

where $v^{j}$ is the velocity flow vector and $\in, \mathrm{p}$ are the density and pressure of the fluid respectively.

The equation for tilted cosmological models is more complicated than those of non-tilted one. The general dynamics of tilted model have been studied by King and Ellis [2], Ellis and King [3], Collin and Ellis [4].Mukherjee [5] has investigated tilted Bianchi type I universe with heat flux in general relativity. He has shown that the universe assumes a pancake shape. The velocity vector is not geodesic and heat flux is comparable to energy density.

Manuscript received May 6, 2012; revised June 18, 2012.

Rakeshwar Purohit is with M. L. Sukhadia University, Udaipur-313001, India (e-mail: ramkrishnadr@gmail.com).

Anita Bagora is with Jaipur National University, Jaipur-302025, India (e-mail: anitabagora@gmail.com).
In general relativity, a dust solution is an exact solution of the Einstein field equation in which the gravitational field is produced entirely by the mass, momentum and stress density of a perfect fluid which has positive mass energy density but vanishing pressure. Concerning the tilted perfect fluid models, Bradly [6] obtained all tilted and expanding dust self similar cosmologies that are hypersurface homogeneous. Carr [7] classified spherically symmetric self similar dust models. Bagora et al. [8]-[9] have investigated tilted dust magnetic cosmological models.

The majority of the studies in cosmology involve a perfect fluid. However, observed physical phenomena such as the large entropy per baryon and the remarkable degree of isotropy of the cosmic microwave background radiation suggests analysis of dissipative effect in cosmology. Furthermore, there are several processes which are expected to give rise to viscous effects. A realistic treatment of the problem requires the consideration of material distribution other than the perfect fluid. Misner [10]-[11] has studied the effect of viscosity on the evolution of cosmological models. Bulk viscosity is associated with the GUT phase transition and strong creation. Also, the introduction of bulk viscosity can avoid the big-bang singularity. The presence of viscosity in the fluid content introduces many interesting features in the dynamics of homogeneous cosmological models [12], [13]. Banerjee et al. [14] have investigated Bianchi-I cosmological models consistency of a fluid with both bulk and shear viscosity. The effect of bulk viscosity on the cosmological evolution has been investigated by a number of authors in framework of general theory of relativity (Padmanabhan and Chitre [15]; Johri and Sudarshan [16]; Maartens [17]; Zimdahl [18]; Kalyani and Singh [19]; Singh, Beesham and Mbokazi [20]. Motivated by these studies,in this paper, we have investigated Bianchi type I tilted dust fluid cosmological model in the presence and absence of bulk viscosity. To get a determinate solution, we have also assumed a supplementary condition $\mathrm{A}=\mathrm{BC}$ between metric potential. The various physical and geometrical aspects of the models are discussed.

\section{The METRIC AND FIELD EQUATIONS}

We consider metric in the form

$$
\mathrm{ds}^{2}=-\mathrm{dt}^{2}+A^{2} \mathrm{dx}^{2}+B^{2} \mathrm{dy}^{2}+C^{2} \mathrm{dz}^{2},
$$

where $\mathrm{A}, \mathrm{B}$ and $\mathrm{C}$ are functions of ' $\mathrm{t}$ ' alone.

The energy momentum tensor for perfect fluid distribution with heat conduction given by Ellis [21] and for bulk viscosity given by Landau and Lifshitz [22] is given by

$$
T_{i}^{j}=(\in+p) v_{i} v^{j}+p g_{i}^{j}+q_{i} v^{j}+v_{i} q^{j}-\zeta \theta\left(g_{i}^{j}+v_{i} v^{j}\right)
$$


Together with

$$
\begin{gathered}
g_{i j} v_{i} v^{j}=-1, \\
q_{i} q^{j}>0, \\
q_{i} v^{i}=0,
\end{gathered}
$$

where $\mathrm{p}$ is the isotropic pressure, $\in$ the matter density and $q_{i}$ the heat conduction vector orthogonal to $v^{i}$. The fluid flow vector has the components $\left(\frac{\sinh \lambda}{\mathrm{A}}, 0,0, \cosh \lambda\right)$ satisfying

(3) and $\lambda$ is the tilt angle.

The Einstein field equation

$$
R_{i}^{j}-\frac{1}{2} R g_{i}^{j}=-8 \pi T_{i}^{j}
$$

(units such that $\mathrm{c}=\mathrm{G}=1$ )

For the line element (1) are

$$
\begin{aligned}
& \frac{B_{44}}{B}+\frac{C_{44}}{C}+\frac{\mathrm{B}_{4} C_{4}}{\mathrm{BC}}=-8 \pi\left[(\in+p) \sinh ^{2} \lambda+p+2 q_{1} \frac{\sinh \lambda}{A}-K \cosh ^{2} \lambda\right] \\
& \frac{A_{44}}{A}+\frac{C_{44}}{C}+\frac{\mathrm{A}_{4} C_{4}}{\mathrm{AC}}=-8 \pi(\in-K) \\
& \frac{A_{44}}{A}+\frac{B_{44}}{B}+\frac{\mathrm{A}_{4} B_{4}}{\mathrm{AB}}=-8 \pi(\in-K)
\end{aligned}
$$

$\frac{A_{4} B_{4}}{A B}+\frac{A_{4} C_{4}}{A C}+\frac{B_{4} C_{4}}{\mathrm{BC}}=-8 \pi\left[-(\in+p) \cosh ^{2} \lambda+p-2 q_{1} \frac{\sinh \lambda}{A}-K \sinh ^{2} \lambda\right]$

$$
\begin{aligned}
& (\in+p) A \sinh \lambda \cosh \lambda+ \\
& q_{1} \cosh \lambda+q_{1} \frac{\sinh ^{2} \lambda}{\cosh \lambda}-K \sinh \lambda \cosh \lambda=0
\end{aligned}
$$

where the suffix ' 4 ' stands for ordinary differentiation with respect to cosmic time ' $t$ ' alone.

\section{SOlution OF THE FIELD EQUATIONS}

Equations from (6) to (10) are five equations in seven unknown $\mathrm{A}, \mathrm{B}, \mathrm{C}, \in, p, \lambda$ and $q_{1}$, therefore to determine the complete solution we assume that the model is filled with dust of perfect fluid which leads to

$$
p=0 \text {. }
$$

To get the deterministic solution we assume a special relation between the metric potentials $\mathrm{A}, \mathrm{B}$ and $\mathrm{C}$ as

$$
\mathrm{A}=\mathrm{BC} \text {. }
$$

Also, we assume that

$$
\zeta \theta=\mathrm{K}
$$

The condition $\zeta \theta=\mathrm{K}$ is due to the peculiar characteristic of the bulk viscosity. It acts like a negative energy field in an expanding universe (Johri and Sudharshan [23]) i.e $\zeta \theta=\mathrm{K}$. According to that expansion is inversely proportional to bulk viscosity. Equations (6) and (9) with (11) lead to

$$
\begin{aligned}
& \frac{B_{44}}{B}+\frac{C_{44}}{C}+\frac{2 \mathrm{~B}_{4} \mathrm{C}_{4}}{\mathrm{BC}} \\
& +\frac{\mathrm{A}_{4} \mathrm{C}_{4}}{\mathrm{AC}}+\frac{\mathrm{A}_{4} \mathrm{~B}_{4}}{\mathrm{AB}}=8 \pi(\in+K)
\end{aligned}
$$

Equations (7) and (8) lead to

$$
\frac{\mathrm{B}_{44}}{\mathrm{~B}}-\frac{\mathrm{C}_{44}}{\mathrm{C}}+\frac{\mathrm{A}_{4}}{\mathrm{~A}}\left(\frac{\mathrm{B}_{4}}{\mathrm{~B}}-\frac{\mathrm{C}_{4}}{\mathrm{C}}\right)=0 \text {. }
$$

This leads to

$$
\frac{v_{4}}{v}=\frac{a}{\mu^{2}}
$$

where $\mathrm{BC}=\mu, \frac{\mathrm{B}}{\mathrm{C}}=v$ and ' $\mathrm{a}$ ' is constant of integration.

Again from equations (7) and (8) with (11), we have

$$
\frac{2 A_{44}}{A}+\frac{B_{44}}{B}+\frac{C_{44}}{C}+\frac{\mathrm{A}_{4} \mathrm{C}_{4}}{\mathrm{AC}}+\frac{\mathrm{A}_{4} \mathrm{~B}_{4}}{\mathrm{AB}}=16 \pi K
$$

Equation (17) leads to

$$
6 \frac{\mu_{44}}{\mu}+\left(\frac{\mu_{4}}{\mu}\right)^{2}+\left(\frac{v_{4}}{v}\right)^{2}=16 \pi \mathrm{K},
$$

where $\mathrm{A}=\mu$.

From equations (16) and (18), we have

$$
2 \mu_{44}+\frac{\mu_{4}^{2}}{\mu}=\frac{-a^{2}}{\mu^{3}}+\frac{16 \pi K \mu}{3}
$$

Equation (19) gives

$$
2 f f^{1}-\frac{f^{2}}{3 \mu}=\frac{-a^{2}}{3 \mu^{3}}+\frac{16 \pi K \mu}{3}
$$

where $\mu_{4}=\mathrm{f}(\mu)$.

Equation (20) leads to

$$
\mu_{4}^{2}=\frac{1}{35 \mu^{7 / 3}}\left[7 a^{2} \mu^{1 / 3}+35 b \mu^{2}+80 \pi K \mu^{13 / 3}\right]
$$

where ' $b$ ' is a constant of integration.

$$
\log \nu=\mathrm{a} \sqrt{35} \int \frac{\mathrm{d} \mu}{\mu^{5 / 6} \sqrt{7 a^{2} \mu^{1 / 3}+35 b \mu^{2}+80 \pi K \mu^{13 / 3}}} .
$$

Hence the metric (1) reduces to the form

$$
\mathrm{ds}^{2}=-\frac{\mathrm{d} \mu^{2}}{\mathrm{f}^{2}}+\mu^{2} d x^{2}+\mu v d y^{2}+\frac{\mu}{v} d z^{2}
$$

where $v$ is determined by (22).

By introducing the following transformations

$$
\mu=T, \quad x=X, \quad y=Y, \quad z=Z .
$$

The metric (23) reduces to the form 


$$
\begin{aligned}
& \mathrm{ds}^{2}=\left[\frac{-35 T^{7 / 3}}{7 a^{2} T^{1 / 3}+35 b T^{2}+80 \pi K T^{13 / 3}}\right] \mathrm{dT}^{2} \\
& +T^{2} d X^{2}+\mathrm{T} v \mathrm{dY} \mathrm{Y}^{2}+\frac{\mathrm{T}}{v} d Z^{2}
\end{aligned}
$$

where $v$ is determined by (23) with $\mu=\mathrm{T}$.

In the absence of bulk viscosity i.e. when $\mathrm{K} \rightarrow 0$, the metric (24) becomes

$$
\mathrm{ds}^{2}=\left[\frac{-35 T^{7 / 3}}{7 a^{2} T^{1 / 3}+35 b T^{2}}\right] \mathrm{dT}^{2}+T^{2} d X^{2}+\mathrm{T} v \mathrm{dY} \mathrm{Y}^{2}+\frac{\mathrm{T}}{v} d Z^{2}
$$

where $v$ is determined by (22) with $\mu=T$ and $K=0$.

\section{SOME PhySICAL AND GEOMETRICAL FEATURES}

The density for the model (24) is given by

$$
8 \pi \in=\frac{35 \mathrm{~b}-24 \pi \mathrm{KT}^{7 / 3}}{42 \mathrm{~T}^{7 / 3}}
$$

The tilt angle is given by

$$
\cosh \lambda=\frac{1}{2} \sqrt{\frac{105 b-432 \pi K T^{7 / 3}}{35 b+24 \pi K T^{7 / 3}}}
$$

The reality conditions

$$
\begin{aligned}
& \text { (i) } \in+p>0, \\
& \text { lead to } \frac{35 b}{24 \pi \mathrm{K}}>0 .
\end{aligned}
$$

The scalar of expansion $\theta$ calculated for the flow vector $v^{j}$ is given by

$$
\theta=\frac{\psi_{1}}{4 T^{13 / 6}} \sqrt{\frac{7 a^{2} T^{1 / 3}+35 b T^{2}+80 \pi K T^{13 / 3}}{35\left(105 b-432 \pi K T^{7 / 3}\right)\left(35 b+24 \pi K T^{7 / 3}\right)^{3}}}
$$

The components of fluid flow vector $v^{i}$ and heat conduction vector $q^{i}$ for the model (24) are given by

$$
v^{4}=\frac{1}{2} \sqrt{\frac{105 b-432 \pi K T^{7 / 3}}{35 b+24 \pi K T^{7 / 3}}}
$$

$q^{4}=\frac{-\left(35 b+528 \pi K T^{7 / 3}\right)\left(35 b-312 \pi K T^{7 / 3}\right)}{6720 \pi T^{7 / 3}\left(7 b-96 \pi K T^{7 / 3}\right)} \sqrt{\frac{105 b-432 \pi K T^{7 / 3}}{35 b+24 \pi K T^{7 / 3}}}$

The non-vanishing components of shear tensor $\left(\sigma_{\mathrm{ij}}\right)$ and rotation tensor $\left(\omega_{\mathrm{ij}}\right)$ are given by

$$
\sigma_{11}=\frac{\psi_{1}}{2 T^{1 / 6}} \sqrt{\frac{\left(7 a^{2} T^{1 / 3}+35 b T^{2}+80 \pi K T^{13 / 3}\right)\left(105 b-432 \pi K T^{7 / 3}\right)}{35\left(35 b+24 \pi K T^{7 / 3}\right)^{5}}}
$$

$\sigma_{22}=\frac{-\nu\left[\psi_{1} T \sqrt{\left(7 a^{2} T^{1 / 3}+35 b T^{2}+80 \pi K T^{13 / 3}\right)}-3 \sqrt{35} a T^{7 / 6} \psi_{2}\right]}{12 T^{13 / 6} \sqrt{35\left(105 b-432 \pi K T^{7 / 3}\right)\left(35 b+24 \pi K T^{7 / 3}\right)^{3}}}$,

$$
\sigma_{33}=\frac{-\left[\psi_{1} \mathrm{~T} \sqrt{\left(7 \mathrm{a}^{2} \mathrm{~T}^{1 / 3}+35 \mathrm{bT} \mathrm{T}^{2}+80 \pi \mathrm{KT}^{13 / 3}\right)}+3 \sqrt{35} \mathrm{aT}^{7 / 6} \psi_{2}\right]}{12 v \mathrm{~T}^{13 / 6} \sqrt{35\left(105 \mathrm{~b}-432 \pi \mathrm{KT}^{7 / 3}\right)\left(35 \mathrm{~b}+24 \pi \mathrm{KT}^{7 / 3}\right)^{3}}}
$$

$\sigma_{44}=\frac{-\left(35 b+528 \pi K T^{7 / 3}\right) \psi_{1}}{6 T^{13 / 6}} \sqrt{\frac{7 a^{2} T^{1 / 3}+35 b T^{2}+80 \pi K T^{13 / 3}}{35\left(105 b-432 \pi K T^{7 / 3}\right)\left(35 b+24 \pi K T^{7 / 3}\right)^{5}}}$

Here

$$
\begin{gathered}
\psi_{1}=3675 \mathrm{~b}^{2}-10368 \pi^{2} \mathrm{~K}^{2} \mathrm{~T}^{14 / 3}-53760 \pi \mathrm{KbT}^{7 / 3}, \\
\psi_{2}=3675 \mathrm{~b}^{2}-10368 \pi^{2} \mathrm{~K}^{2} \mathrm{~T}^{14 / 3}-12600 \pi \mathrm{KbT}^{7 / 3} .
\end{gathered}
$$

The rates of expansion $\mathrm{H}_{\mathrm{i}}$ in the direction of $x, y$ and $z$ axes are given by

$$
\mathrm{H}_{1}=\frac{1}{\mathrm{~T}^{13 / 6}} \sqrt{\frac{\left(7 \mathrm{a}^{2} \mathrm{~T}^{1 / 3}+35 \mathrm{bT} \mathrm{T}^{2}+80 \pi \mathrm{KT}^{13 / 3}\right)}{35}},
$$

$$
H_{2}=\frac{1}{2 T^{13 / 6}} \sqrt{\frac{\left(7 a^{2} T^{1 / 3}+35 b T^{2}+80 \pi K T^{13 / 3}\right)}{35}}+\frac{\mathrm{a}}{2 \mathrm{~T}^{2}},
$$

$$
H_{3}=\frac{1}{2 T^{13 / 6}} \sqrt{\frac{\left(7 a^{2} T^{1 / 3}+35 b T^{2}+80 \pi K T^{13 / 3}\right)}{35}}-\frac{\mathrm{a}}{2 \mathrm{~T}^{2}} \text {. }
$$

\section{DISCUSSION}

The model starts with a big-bang at $\mathrm{T}=0$ and the expansion in the model decrease as time $\mathrm{T}$ increases and it stops at $\mathrm{T}=\infty$. The model has point type singularity at $\mathrm{T}=$ 0 (MacCallum[24] ). The model represents shearing and expanding universe in general .Since $\operatorname{Lim}_{\mathrm{T} \rightarrow \infty} \frac{\sigma}{\theta} \neq 0$, then the model does not approach isotropy for large value of $\mathrm{T}$. Density $\in \rightarrow 0$ as $\mathrm{T} \rightarrow \infty$ and $\in \rightarrow \infty$ as $\mathrm{T} \rightarrow 0$. When $\mathrm{T}$ $\rightarrow 0 . \mathrm{q}^{4} \rightarrow \infty$ and $\mathrm{q}^{4}$ etend to zero as $\mathrm{T} \rightarrow 0$. At $\mathrm{T}=0$, the Hubble parameters tend to infinite at the time of initial singularity of vanish as $\mathrm{T} \rightarrow \infty$. The reality conditions $\in+$ $\mathrm{p}>0, \in+3 \mathrm{p}>0$ given by Ellis [25] are satisfied when $\mathrm{T}^{7 / 3}>\frac{35 \mathrm{~b}}{24 \pi \mathrm{K}}$.

In the absence of bulk viscosity, $\in \rightarrow \infty$ when $\mathrm{T} \rightarrow 0$ and $\in \rightarrow 0$ when $\mathrm{T} \rightarrow \infty$, therefore $\in$ is the decreasing function of time. At the initial stage the tilt angle is given by $\cosh \lambda=\sqrt{3} / 2$ and at $\mathrm{T}=\infty$, the model reduces to a nontilted universe in nature and fluid distribution tends to be comoving. the model an expanding universe in which the lines of flow of matter are geodesic, shearing . 


\section{REFERENCES}

[1] M. P. R. Jr and L. C. Shepley, "Homogeneous Relativistic Cosmologies," Princeton University Press, Princeton, New Jersy. 1975.

[2] A. R. King and G. F. R. Ellis, Comm. Math. Phys, vol. 31, no. 209, 1973.

[3] G. F. R. Ellis and A. R. King, Comm. Math. Phys, vol. 38, pp. 119, 1974.

[4] C. B. Collins and G. F. R. Ellis, Phys. Rep, vol. 56, no. 65, 1979.

[5] G. Mukherjee. J. Astrophys. Astron, vol. 4, no. 295, 1983.

[6] M. Bradley. Class. Quantum Gravity, vol. 5, no. L15, 1988.

[7] B. J. Carr, Phys. Rev, pp. 044022, 2000

[8] A. Bagora, Turk. J. Phys. vol. 33, pp. 1-11. 2009.

[9] A. Bagora, Adv. Studies Theor. Phys, vol. 2, no. 17, pp. 817-824. 2008.

[10] C.W. Misner, Nature, vol. 214, pp. 40-41, 1967

[11] C.W. Misner, Astrophys. J, vol. 151, pp. 431-457, 1968.

[12] C. B. Collins and J. M. Stewart, Mon. Not. Roy. Astron. Soc, vol. 153, pp. 419-434, 1985.

[13] J. D. Nightingale, Astrophys. J, vol. 185, pp.105-114, 1973.

[14] A. Banerjee, S. B. Duttachoudhary, and A. K. Sanyal, J. Math. Phys, vol. 26, no. 11, pp. 3010-3015, 1985.
[15] T. Padmanabhan and S. M. Chitre, Phys. Lett, pp. 433-436, 1987.

[16] V. B. Johri, and R. Sudarshan, Phys. Lett, pp. 316-320, 1988.

[17] R. Maartens, Class. Quantum Gravit, vol. 12, no. 6, pp. 1455-1465. 1995.

[18] W. Zimdahl, Phys. Rev, pp. 5483-5493, 1996.

[19] D. Kalyani, G. P. Eds, V. d. S. Singh, and T. Singh, Bulk Viscous Models in Lyra's Geometry; New Directions in Relativity and Cosmology, Hadronic Press, USA, pp. 41-47,1997.

[20] T. Singh, A. Beesham, and W.S. Mbokazi, Gen. Relativ. Gravit, vol. 30, no. 4, pp. 537-581, 1998.

[21] G. F. R. Ellis, General Relativity and Cosmology edited by R.K Sachs, Academic Press, New York, pp. 116, 1971.

[22] L. D. Landau and E. M. Lifshitz, Fluid Mechanics; Pergamon Press; vol. 6 , no. 505, 1963.

[23] V. B. Johri and R. Sudarshan, in Proc. Int. Conf. on Mathematical Modelling in Science and Technology, World Scientific, Singapore, 1988.

[24] M. A. H. MacCallum, Comm. Maths. Phys, vol. 20, no. 57, 1971.

[25] G. F. R. Ellis, General Relativity and Cosmology edited by R.K. Sachs, Academic Press; New York, pp.117, 1971. 